\title{
PINTURAS REFORZADAS CON HILOS CORTOS DE VIDRIO
}

\section{(SHORT CUT GLASS YARN REINFORCED COATINGS FOR BUILDING APPLICATIONS)}

Carmen Pérez González, Prof. Asociada. U. C. M.

Pablo Ignacio Comino Almenara, Investigador de Saint Gobain. Vetrotex Cem-FIL, S. L.

Raquel Sánchez Camacho, Investigador de Saint Gobain. Vetrotex Cem-FIL, S. L.

Blanca Parga Landa, Prof. Titular, U. P. M.

Francisco Hernández Olivares, Catedrático, U. P. M.

Fecha de recepción: 24 IV-03

ESPAÑA

$671-6$

\begin{abstract}
RESUMEN
La presente investigación estudia la viabilidad del empleo de refuerzos de fibra de vidrio para pinturas de interior $y$ exterior con el fin de paliar parte de las patologías observadas en aplicaciones arquitectónicas. Se ha seleccionado un tipo de fibra de refuerzo dispersable y se ha estudiado el comportamiento de tres tipos de pinturas (temple, plástica y de impermeabilización) para fracciones gravimétricas crecientes de refuerzo y distintos procesos de aplicación de la pintura.
\end{abstract}

Se ha conseguido mejorar las propiedades de las pinturas industriales que existen en el mercado para aplicaciones interiores y exteriores en edificación elevando las propiedades mecánicas y de durabilidad de dichas pinturas, sin que ello suponga ningún cambio significativo en las cualidades de la pintura, como son la colorimetría, el brillo y el espesor de pelicula.

Palabras clave: pinturas industriales, fibra de vidrio, pintura al temple reforzado, pintura plástica reforzada, pintura de impermeabilización reforzada.

\section{SUMMARY}

Current research literature on coatings for building applications make evident the existence of important damage both in outdoors and indoors applications when the paint is used without any reinforcement because of several reasons: the low quality of some paint components, coatings extremely thin, and the unskilful of painters.

This article studies the behaviour of some industrial paints for indoors and outdoors applications when reinforced with different gravimetric fractions of dispersable short cut glass fibre yarns. It is also studied the support influence (cement mortar, plasterboard and laminated plasterboard) and the application method (handy or sprayed). A better performance and durability is obtained for the fractions by weight which are determined for each type of paint, support and application method. Neither the paint colour properties nor the brightness and texture get worse when these gravimetric fractions of glass fibre are added to the paint.

Key words: paint for buildings, glass fibre, distemper, organic polymer paint, water-proof paint, reinforced paint.

\section{INTRODUCCIÓN}

Los estudios actuales sobre los revestimientos de pintura utilizados en edificación ponen de manifiesto la aparición de patologías diversas en aplicaciones de pinturas de interior y exterior. Los problemas detectados tienen diversas causas entre las que se encuentran: la disminución de la calidad de las pinturas debido a las materias primas empleadas en su fabricación, la aplicación de escasos es- pesores de pintura o, la cada vez mayor falta de profesionales especializados en la aplicación de pinturas especiales. Estos factores, entre otros, provocan que los mencionados daños sean cada vez más frecuentes.

El Grupo Saint Gobain por medio de su filial Vetrotex CemFIL S.L. en su búsqueda para proveer al mercado de nuevos materiales y técnicas innovadoras aplicables en la arquitectura, se ha adentrado en el mundo de las pinturas de 
interior y exterior sobre diversos soportes. Y ante la gama de patologías detectadas en las pinturas de exterior e interior sin reforzar ha querido estudiar, como posible solución, el refuerzo de dichas pinturas con hilos cortos de vidrio.

Las fibras que se han utilizado en este estudio, fabricadas por Saint Gobain Vetrotex, son fibras de característica dispersable $\mathrm{y}$, tal como se describirá mas adelante, tienen un funcionamiento distinto del que presentan los hilos integros, que son los mas utilizados para el refuerzo de materiales. Las fibras de característica dispersable empleadas presentan unas importantes propiedades de refuerzo tanto en medios ácidos como alcalinos, lo que las hacen sumamente interesantes para el refuerzo de pinturas ya que éstas presentan todo tipo de $\mathrm{pH}$ siendo éste dependiente de los materiales empleados en su fabricación, tipo, de la aplicación final y de los ambientes y soportes donde vaya a ser colocada la pintura.

La investigación que se presenta en este artículo se centra fundamentalmente en el estudio de la viabilidad de este tipo de refuerzo para pinturas de interior y exterior, aplicadas con distintos procedimientos, y sobre distintos tipos de soporte.

En un principio se describen los objetivos del proyecto, el tipo de pinturas que se han seleccionado, se describe la fibra de refuerzo que se propone como refuerzo y el modo en el que opera, se describen también los soportes que se han seleccionado y los métodos de aplicación.

El estudio de la viabilidad de este tipo de refuerzo en una pintura cuyo uso se centra en el ámbito arquitectónico y de la edificación precisa definir aquellos aspectos que se considera que son determinantes para tal tipo de aplicaciones. Y en el ámbito arquitectónico se han definido como aspectos determinantes los siguientes: el acabado del producto, las cualidades de la pintura asociadas, como son la colorimetría, el brillo y el espesor de película, y el comportamiento a largo plazo frente a los agentes externos. Estos aspectos determinan, a su vez, otra característica que interesa a nuestro estudio como es, por ejemplo, la dosificación de refuerzo, es decir, la determinación de la proporción más adecuada de fibra para estos tipos de aplicaciones.

A continuación se describen, también, los ensayos realizados. Y, finalmente, las conclusiones alcanzadas.

\section{OBJETIVOS}

El proyecto se ha centrado en los siguientes objetivos:

- Definir los tipos de pintura, de procedimientos de aplicación y de soportes objeto de estudio.
- Realizar un estudio comparativo de algunas propiedades organolépticas (fundamentalmente propiedades visuales) de las diferentes pinturas de interior y exterior seleccionadas, reforzadas con porcentajes variables de fibra de vidrio y compararlas con las que exhiben las pinturas sin reforzar. Todo ello utilizando los diversos procedimientos de aplicación que se han definido en el proyecto y los soportes definidos como objeto de estudio en este proyecto.

- Analizar las propiedades de las pinturas seleccionadas cuando se les añade en estado fresco bajas dosificaciones de fibra de vidrio. Caracterizar los productos obtenidos tras someterlos a ciclos en cámaras climáticas y radiación ultravioleta. Determinar las propiedades que se han definido como determinantes de una aplicación arquitectónica: brillo, color, espesor de película y defectos.

- Realizar un estudio comparativo de la durabilidad de las pinturas objeto de estudio con o sin refuerzo de hilos de vidrio, analizando también sus prestaciones a largo plazo.

- Determinar las cantidades óptimas de refuerzo de fibra de vidrio en las diferentes aplicaciones seleccionadas.

\section{MATERIALES}

\subsection{Pinturas. Procedimientos de aplicación}

Las pinturas que se han seleccionado para este estudio han sido pinturas comerciales al temple, pintura plástica y pintura de impermeabilización. Las marcas comerciales con las que se ha trabajado pertenecen a las empresas Colamina, Valentine y Cepisa.

Se han utilizado los procedimientos más usuales para la aplicación de la pintura, como son: la brocha, el rodillo y el compresor con pistola.

Como planteamiento inicial, en todos los procedimientos y en todos los casos se ha trabajado con un único color.

\subsection{Fibra de vidrio}

El hilo de vidrio utilizado es de fibra Cem-FIL suministrado por Saint Gobain Vetrotex S.A. Se trata de una fibra resistente a los ácidos y a los álcalis, de muy altas prestaciones.

La fibra utilizada es de característica dispersable, característica que se explica a continuación.

Es bien conocido que una de las principales características de las fibras de vidrio de refucrzo es su formato en forma de haz de filamentos que, unidos entre sí, forman un hilo de vidrio llamado "hilo de base". Este "hilo de base" se define por su densidad lineal -también denomi- 
nada a veces formato-, que, de acuerdo con la norma ASTM (D-861), se mide en una unidad llamada Tex. El número de Tex se define como el peso expresado en gramos de 1.000 metros de hilo. Por lo tanto, tanto la densidad lineal, el número de Tex o el formato de un hilo dependen del diámetro y del número de los filamentos que lo constituyen.

Se dice que un "hilo de base" es un "hilo íntegro" cuando el formato, el número de Tex o la densidad lineal del hilo es idéntica, antes y después de la trasformación. Este tipo de hilo es el más conocido y el más utilizado a nivel mundial en el refuerzo de materiales. En efecto, en las aplicaciones convencionales en las que se utilizan fibras de vidrio como material de refuerzo de una matriz para formar un material compuesto de mayores prestaciones, el formato de hilo de base no se pierde con la aplicación, esto es, el hilo de base de refuerzo -formado por multitud de filamentos-, se mantiene exactamente igual -con idéntica densidad lineal o número de Tex-, antes y después de su mezcla con la matriz y el proceso de transformación que genera el material compuesto. De esta manera, los haces de filamentos que constituyen el hilo de base trabajan conjuntamente para la consecución de las altas prestaciones mecánicas que presentan los materiales compuestos reforzados con fibras de vidrio $\mathrm{E}$.

Pero en el caso del presente estudio, el hilo de refuerzo que se ha utilizado es muy diferente al descrito como íntegro ya que es un hilo de altas prestaciones que pertenece a la familia de productos dispersables.

Este tipo de hilo de base presenta un comportamiento distinto del hilo integro ya que una vez incorporado a la matriz pierde su formato: se produce la apertura del mismo en los filamentos que lo constituyen y se dispersa.

Esta dispersión del hilo de base es facilitada por la acción de un ensimaje especial con el que se recubre cada uno de los filamentos constituyentes del hilo de base.

Como el hilo de base está constituido por un haz de cientos de filamentos, una vez abierto o dispersado el hilo de base en la matriz -en este caso la pintura-, la acción de refuerzo la van a realizar los filamentos que constituían el hilo; filamentos que, a su vez, tienen un nuevo formato, número de Tex o densidad lineal preciso e idéntico entre ellos.

Dado que la fibra o hilo de base que se ha empleado en el presente estudio ha de tener una longitud determinada (entre los 3 y $6 \mathrm{~mm}$ de longitud dependiendo de las prestaciones requeridas, de los soportes, de los espesores de película y, sobre todo, del propio proceso de aplicación), y que cada hilo de base se compone de más de 800 filamentos constituyentes, una vez realizada la mezcla y haberse producido la dispersión de los hilos de base en los fila- mentos que los componen, el refuerzo conferido a la pintura está basado en multitud de filamentos de aproximadamente 14 micras de diámetro cada uno, aleatoriamente distribuidos, que forman una red tupida de refuerzo de la pintura.

En la Tabla 1, se relacionan la formulación de un hilo de vidrio $\mathrm{E}$ (de escaso potencial en esta aplicación de refuerzo de pinturas, pero de mayor difusión y conocimiento en el mercado general del refuerzo con fibras de vidrio) y la formulación del Hilo de Refuerzo Cem-FIL Ácido-Álcali resistente que se ha empleado en la presente investigación.

TABLA 1

Comparación de las formulaciones de fibras de vidrio tipo común $\mathrm{E}$ y tipo $\mathrm{AR}$.

\begin{tabular}{|lcc|}
\hline \multicolumn{1}{|c|}{ Componente } & $\begin{array}{c}\text { Vidrio-E } \\
(\%)\end{array}$ & $\begin{array}{c}\text { Vidrio-AR } \\
\text { Cem-FIL } \\
(\%)\end{array}$ \\
\hline $\mathrm{SiO}_{2}$ & 54.0 & 62.0 \\
$\mathrm{Na}_{2} \mathrm{O}$ & & 14.8 \\
$\mathrm{CaO}$ & 22.0 & 5.6 \\
$\mathrm{MgO}$ & 0.5 & \\
$\mathrm{~K}_{2}$ & 0.8 & \\
$\mathrm{Al}_{2} \mathrm{O}_{3}$ & 15.0 & 0.8 \\
$\mathrm{Fe}_{2} \mathrm{O}_{3}$ & 0.3 & \\
$\mathrm{~B}_{2} \mathrm{O}_{3}$ & 7.0 & 16.7 \\
$\mathrm{ZrO}_{2}$ & & 0.1 \\
$\mathrm{TiO}_{2}$ & & \\
$\mathrm{Li}_{2} \mathrm{O}$ & & \\
\hline
\end{tabular}

En lo que a dosificaciones de refuerzo se refiere el planteamiento inicial fue el de obtener pinturas reforzadas con el mayor porcentaje posible de fibra, cumpliendo siempre los requisitos de comportamiento marcados por el proyecto.

\subsection{Soportes}

Los soportes sobre los que han sido aplicadas las pinturas reforzadas y sin reforzar con los métodos de aplicación seleccionados (brocha, rodillo y compresor con pistola), han sido los siguientes:

- Mortcro de cemento (Cem II A/V 42,5 R) para aplicaciones en exteriores.

- Soporte industrial de cartón-yeso de 12,5 mm de espesor normal para aplicaciones interiores. 
- Yeso fino (escayola) de la marca San Martín, tipo E30 para aplicaciones interiores.

\section{PLAN DE ENSAYOS}

\subsection{Confección de probetas}

Para la aplicación con brocha y rodillo se ha trabajado sobre probetas realizadas a pequeña escala $(12,5 \times 4,5 \mathrm{~cm})$, con el objeto de que se puedan ensayar fácilmente en laboratorio dichas probetas. El soporte se preparó con una capa de acetato de polivinilo previa a la aplicación de la pintura.

Para la aplicación de la pintura a pistola se han realizado probetas de mayor tamaño $(30 \times 60 \mathrm{~cm})$, de forma que permitiese una mayor facilidad para la proyección y que reflejase un acabado más real. Los soportes fueron reforzados con el mismo tipo de fibra de vidrio (excepto el de cartón-yeso, que es industrial) y preparados igualmente con una capa de PVA que se dejó secar antes de aplicar la pintura.

Las pinturas en este caso han sido suministradas por Colamina y fueron aplicadas con compresor de aire "Airlessco".

La denominación de las muestras ha seguido las siguientes normas establecidas para la codificación de las probetas: comienza con un código de soporte-pintura (por ejemplo MT, mortero y temple) y finaliza con el código de procedimiento de aplicación (B-brocha, R-rodillo y P-pistola). Cuando la muestra analizada contiene fibra de refuerzo, se indica entre los códigos anteriores, en primer lugar, la longitud de los hilos (3 ó $6 \mathrm{~mm}$ ) y después el porcentaje en peso de fibra agregado $(0,25 \% ; 0,5 \%$ y $1 \%)$.

\subsection{Primera Fase}

En primer lugar se llevó a cabo el planteamiento de un modelo de utilidad, definiendo el proceso de realización de las pinturas. Además, se efectuó un análisis visual de las probetas para detectar posibles problemas en el comportamiento de las pinturas preparadas. Este primer análisis debía servir para realizar una primera selección de las mezclas más idóneas para efectuar las conclusiones posteriores. Finalmente se seleccionó la longitud de los hilos: de 3 y $6 \mathrm{~mm}$

\subsubsection{Brocha y rodillo}

Con las longitudes seleccionadas para los hilos (3 y $6 \mathrm{~mm}$ ) se probaron diferentes proporciones en peso para reforzar la pintura, obteniendo resultados visualmente válidos con las mezclas de $0,5 \%$ y $1 \%$ de hilos de refuerzo respecto al peso de las pinturas.
Con la adición del $0,5 \%$ en peso de fibra, el resultado visual es óptimo. Sin embargo, para el porcentaje del $1 \%$ se aconseja aumentar el tiempo de batido de la pintura con la fibra para que no se produzcan irregularidades por agrupamientos de fibra en la superficie, sobre todo cuando se utiliza el procedimiento de rodillo.

Los hilos de fibra de $6 \mathrm{~mm}$ de longitud que habían sido inicialmente seleccionados para su utilización en las probetas junto a los de $3 \mathrm{~mm}$, fueron descartados tras los ensayos ya que visualmente se obtenían resultados inaceptables por sus acumulaciones de fibra en superficie.

\subsubsection{Pistola}

Las muestras recibieron dos capas de pintura, obteniéndose, en algunas de ellas, un acabado con fibras que sobresalían de la superficie, lo que hizo necesario rebajarlas utilizando un rodillo.

Los porcentajes de refuerzo con los que se trabajó fueron 0,25 y $0,4 \%$ en peso, ya que, con porcentajes superiores, la pistola se atascaba. La aplicación de la pintura con un $0,4 \%$ de refuerzo ya presentó problemas de obstrucción en el compresor de pintura.

Los mejores acabados visuales, sin necesidad aplicar una segunda capa con rodillo, se obtienen en los soportes de mortero con $0,25 \%$ de refuerzo. Por tanto, este soporte se revela como una buena base para este nuevo tipo de pintura, mezcla de fibra y diversas técnicas, ya que tiene un buen acabado y no necesita un aplastado de las posibles fibras levantadas después de la aplicación. En el resto de los soportes se necesita un acabado con rodillo para aplastar las fibras salientes.

En estas pruebas realizadas hay que hacer mención a la inmejorable respuesta de este producto con la pintura de impermeabilización de Colamina, ya que la fibra queda uniformemente distribuida y no se producen levantamientos.

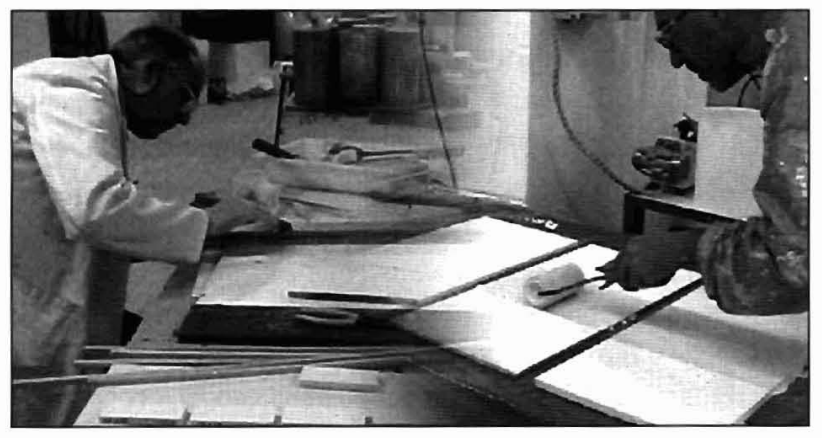

Figura l.- Imágenes del proceso de realización de las probetas. Ala izquierda se ve la preparación de muestras de pequeño tamaño y, a la derecha, de las más grandes. 


\subsection{Segunda Fase}

Realizada esta primera fase de análisis visual, se estaba en disposición de someter las probetas a los ensayos programados. Dichos ensayos fueron realizados en el Centro Tecnológico de la Madera y en el Laboratorio de Materiales de la ETS Arquitectura.

\subsubsection{Ensayos realizados}

Se han analizado las propiedades de la pintura tanto en húmedo como en seco.

Así, para la pintura en estado líquido se han medido la viscosidad y el $\mathrm{pH}$, tanto con diversas cantidades de hilos cortos de vidrio como sin fibra. Las muestras preparadas consistieron en botes de $1,5 \mathrm{~kg}$ de pintura. ${ }^{1}$

Además, se ha explorado el comportamiento reológico de las pinturas sin fibra y con diversas cantidades de hilos cortos de vidrio bien mezclados en su masa, con el objeto de obtener información sobre las mezclas óptimas desde el punto de vista de su aplicabilidad y comportamiento como pintura.

Las propiedades analizadas han sido:

- La adherencia al soporte

- El extendido en superficie

- La homogeneidad en el acabado

- El mezclado adecuado

- El poder cubriente

entre otros conceptos que definen el comportamiento de una pintura en húmedo.

Los análisis realizados con las muestras en estado seco tienen como objeto definir el acabado del producto y determinar la resistencia de las pinturas frente a situaciones que se dan en la vida real. Y para ello se realizaron ensayos de envejecimiento con el fin de examinar los efectos producidos por distintos tipos de ambientes agresivos sobre el acabado.

Así, se realizó una observación visual para analizar no solo los conceptos relacionados anteriormente sino también los posibles ojillos, craquelados superficiales y agrupaciones de fibra.

Se han planteado diversos ensayos de envejecimiento:

- Ensayos en cámara Xenón. Método de ensayo a utilizar para determinar la resistencia al cambio de color de los

' El equipo utilizado ha sido Viscosimetro Brookfiel modelo DV-I+, M/02.040 y el medidor de $\mathrm{pH} / \mathrm{mv} /{ }^{\circ} \mathrm{C}$, microprocesador de sobremesa.

2Procedimiento de ensayo basado en la norma UNE 56.843.01 Apto. 4. Resistencia del color a la luz en cámara de Xenón durante 95 horas. UNE 53-235-85. Métodos de exposición a una fuente de luz artificial. revestimientos superficiales sometidos a la acción de la luz de una lámpara de Xenón².

- Ensayos en cámara niebla salina. Método de ensayo a utilizar para determinar el comportamiento de los revestimientos superficiales sometidos a ambientes salinos $^{3}$.

- Ensayos en cámara climática sometiendo a las probetas a variaciones extremas de humedad y temperatura. Se han seleccionado nueve ciclos tipo, a fin de simular unas condiciones agresivas que puedan producir una alteración similar a la observada en condiciones ambientales en regiones climáticas extremas ${ }^{4}$.

- Ensayos en cámara climática para la resistencia al cuarteamiento. Establece un método para determinar la resistencia al cuarteamiento de pinturas cuando se someten a ciclos de enfriamiento repentino. Se buscan grietas distribuidas al azar, formando una red sobre una parte o totalidad de la probeta, produciendo un aspecto comparable a una fisuración de la película de pintura ${ }^{5}$.

Este procedimiento ha consistido en ciclos de aire a la temperatura de $50^{\circ} \mathrm{C}$ durante 1 hora en cámara climática, seguidos de 1 hora en congelador a $-20^{\circ} \mathrm{C}$. A continuación se lleva a temperatura ambiente durante 15 minutos y se examinan (luz rasante y directa).

El ensayo tiene como objeto comprobar si la muestra soporta un número determinado de ciclos. El número de ciclos que se ha seleccionado ha sido de 14 ciclos (que correspondería a 14 años en la vida real) para las muestras de rodillo y brocha y de diez ciclos para las muestras realizadas con pistola.

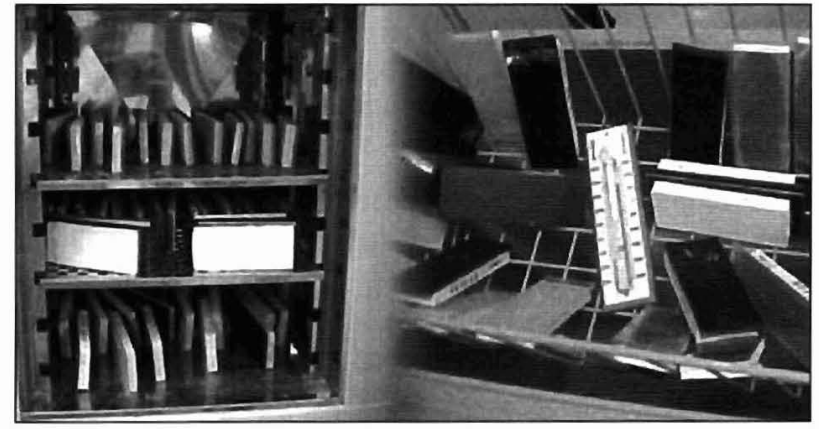

Figura 2.- Dos de las cámaras de envejecimiento utilizadas: cámara climática y congelador industrial.

Las muestras están sometidas a una solución de vapor de agua con $50 \mathrm{~g}$ de sal yodada por litro durante 24 horas. La Cámara de niebla salina utilizada ha sido PHmeter, Balanza, Vaso aforado, Hobbo y la norma utilizada $11-P E N-M B-20$ del Centro Tecnológico de la Madera.

" Cámara climática Modelo $305 \mathrm{SB} /+10 \mathrm{Ju} / 40 \mathrm{Du}$ con el "Test programe" más severo para determinar el comportamiento de las superficies ante variaciones extremas de humedad y temperatura y la norma UNE 56.843.01.

"Según norma UNE 48-025-79 "Resistencia a ciclos de enfriamiento". Se han cuantificado los indices de mejora del comportamiento utilizando las Cámaras Climáticas (modelo CHESA) y el congelador industrial. 
Se ha medido también el espesor de película. Éste se obtuvo fotografiando la pintura lateralmente con una lupa y utilizando un programa de ordenador para tomar valores del espesor de la capa de pintura ${ }^{6}$.

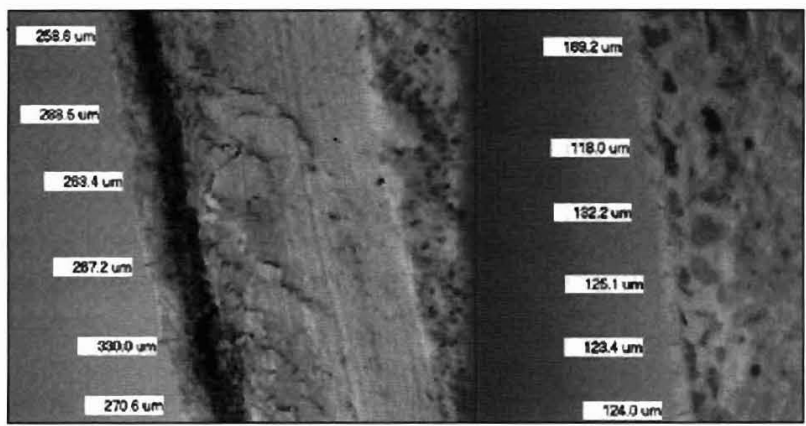

Figura 3.- Dos imágenes del programa de ordenador usado para la medición del espesor de pelicula. A la izquierda, cartón-yeso con pintura plástica y, a la derecha, mortero y temple.

Otros ensayos realizados han sido la determinación de la variación colorimétrica ${ }^{7}$ y del brillo ${ }^{8}$.

La finalidad de los ensayos colorimétricos a los que se sometió la pintura era realizar medidas del color de las muestras a través de las coordenadas de color y del valor triestímulo, que se tomaron antes y después de ciclos de envejecimiento en cámara climática (en este caso cámara Xenotest y de Niebla Salina). Los parámetros iniciales y finales, en relación con la normativa de referencia, indican la posible alteración sufrida.

El tiempo real al que equivale el envejecimiento aplicado es un año. Al tener los dos datos se determinarán las diferencias de color según el sistema Cielab. La finalidad de este ensayo es determinar, mediante un proceso acelerado de envejecimiento, la solidez del color en varias muestras diferentes para poder comparar su estabilidad 9 . Se parte de las coordenadas cromáticas definidas por el sistema cromático Cielab $\left(\mathrm{L}^{*}, \mathrm{a}^{*}\right.$ y $\left.\mathrm{b}^{*}\right)$ y se calculan cuatro parámetros: $\mathrm{DE}$, magnitud de la diferencia de color entre las muestras; DC, diferencia de cromaticidad; DS, diferencia de saturación, y DT, diferencia de tono.

Estos parámetros se determinan con los cálculos siguientes:

$\mathrm{DL}=\mathrm{Lp}-\mathrm{Ls}$

$\mathrm{DE}=\left[(\mathrm{DL})^{2}+(\mathrm{Da})^{2}+(\mathrm{Db})^{2}\right]^{1 / 2}$

"Materiales utilizados: Cámara de video Moticam 480 P y programa de ordenador Motic Images 20001.2

'El equipo utilizado fue Colorímetro triestímulo M/02.039 con procesador de datos DP-30I marca Minolta y las normas han sido: UNE 48.073.94 Parte 2 Apartado 9.2. UNE 40-435-84. Diferencias de color según el sistema Cielab.
$\mathrm{DC}=\left[(\mathrm{ap}-\mathrm{as})^{2}+(\mathrm{bp}-\mathrm{bs})^{2}\right]^{1 / 2}$

$\mathrm{DS}=\left(a p^{2}+b p^{2}\right)^{1 / 2}-\left(a s^{2}+b s^{2}\right)^{1 / 2}$

$\mathrm{DT}=\left(\mathrm{DC}^{2}-\mathrm{DS}^{2}\right)^{1 / 2}$

(donde "p" indica los valores iniciales y "s" los finales)

Para las mediciones del brillo se ha utilizado un ensayo de brillometría cuyo principio está basado en la medida, antes y después de ciclos de envejecimiento en cámara climática, del brillo especular, que es la reflectancia fraccional luminosa de la muestra en la dirección especular. Para medirla se han empleado tres diferentes condiciones geométricas: 20,60 y $85^{\circ}$.

Realizadas las mediciones, se realiza el ensayo comparativo de determinación de la variación del brillo en pinturas sometidas a envejecimiento. El indicador utilizado para la determinación del deterioro producido en la superficie de las muestras ha sido la variación de sus propiedades de reflexión, es decir, del brillo de cada una de ellas.

Después de estos ensayos se han extraído unos resultados para caracterizar el comportamiento de las pinturas sobre distintos soportes, con mayor o menor cantidad de hilos cortos de vidrio, de tal modo que se pueda estimar la cantidad recomendable de hilos en función del tipo de soporte, tipo de pintura y ambiente al que estará sometido.

\section{RESULTADOS}

A continuación se detallan los resultados obtenidos en los diferentes ensayos:

\subsection{Ensayos de resistencia a la congelación- descongelación y de resistencia mecánica (resistencia al cuarteamiento) realizados en cámaras climáticas}

\subsubsection{Procedimiento a pistola}

Todas las muestras realizadas con el procedimiento a pistola, con el soporte reforzado y las proporciones $0,25 \%$ y $0,4 \%$ en peso, se han comportado adecuadamente ante estos ensayos, a excepción de las muestras con soporte de yeso, de las que algunas (con fibra o sin ella) han sufrido la fragmentación de la capa de pintura e incluso del soporte.

Así, la pintura al temple sobre yeso sin fibra ha craquelado al cuarto ciclo mientras que con la incorporación de fibra aguantó un ciclo más, aunque también craqueló.

\footnotetext{
* Equipos utilizados: Brillómetro, patrón de brillo especular e higrómetro. Brillómetro TR i-MiCROGLOSS 20-60-85 marca SHEEN. Norma utilizada UNE 48.026.80 pinturas y barnices. UNE I1-019.89 Parte I'A Brillo Especular. ${ }^{9}$ Determinación de diferencias de color según CIELAB. UNE 40-435-84. Diciembre 1984.
} 
Otra pintura dañada es la pintura plástica sin fibra sobre yeso, craquelada en el primer ciclo. La adición de fibra hace que la pintura sea estable en los 10 ciclos que se realizaron.

En la Tabla 2 se resumen los resultados obtenidos en este ensayo por tipo de soporte y pintura, se ha empleado el símbolo "OK" cuando la probeta pasa el ensayo sin que aparezcan daños, o bien, especificando el número de ciclo en el que aparece el primer daño, que, en este caso, es un craquelado.

Este ensayo demuestra que el soporte es determinante en la estabilidad de las pinturas. También se ha observado que la adición de fibra consigue mejorar la resistencia de las pinturas a ser dañadas por los posibles movimientos del soporte.

\subsubsection{Procedimientos con brocha y con rodillo}

En ambos procedimientos las muestras de pintura plástica sobre yeso mejoran con la adición de fibra. La muestra con una adición del $0,5 \%$ aguanta un ciclo más que la pintura sin fibra y la adición de fibra del $1 \%$ en peso vuelve a la pintura estable durante los 14 ciclos aplicados.

La pintura al temple sobre yeso, aplicada con brocha o con rodillo, también mejora con la adición de fibra. En este tipo de pintura, tan inestable ante la humedad y los cambios bruscos de temperatura, la adición de fibra hace que el procedimiento gane durabilidad de forma directamente proporcional a la cantidad de fibra utilizada. Así, con $0,5 \%$ de fibra aguanta un ciclo más y con el $1 \%$, dos ciclos más.

La pintura plástica sobre yeso laminado en la aplicación con rodillo mejora con la adición de fibra, ya que si se añade el $0,5 \%$ de fibra sobre la pintura plástica se consi- guen dos ciclos más de vida y si se aumenta hasta el $1 \%$, vuelve al procedimiento estable.

Con brocha fueron estables las muestras, tanto con fibra como sin ella.

La pintura al temple sobre yeso laminado mejora igualmente con la adición de fibra. Mientras que la muestra sin fibra realizada con brocha craquela al quinto ciclo y la realizada con rodillo al cuarto, la adición del $0,5 \%$ de fibra en peso le da un ciclo más en el procedimiento a brocha y 9 ciclos más con el procedimiento a rodillo. Con un porcentaje de fibra del 1\%, la pintura se hace estable en ambos procedimientos de aplicación.

La pintura plástica sobre mortero sin la adición de fibra resiste 12 ciclos en el procedimiento a rodillo, mientras que la adición de fibra vuelve estable la pintura (14 ciclos). Con brocha fueron estables tanto las muestras con fibra como sin ella.

La pintura al temple sobre mortero, con el procedimiento a rodillo y sin fibra, rompe al quinto ciclo; y al octavo ciclo la pintura está totalmente levantada del soporte. En este procedimiento la adición de fibra dota a la pintura de estabilidad.

Con el procedimiento a brocha, sin fibra rompe al octavo y se levanta en el ciclo decimotercero. Añadiéndole fibra al $0,5 \%$ en peso se obtiene un ciclo más y la adición de un $1 \%$ dota a la pintura de estabilidad.

La pintura de impermeabilización sobre mortero sin fibra no resiste más que un ciclo en cualquiera de los dos procedimientos. Con la adición de fibra mejora de forma que, con el $0,5 \%$, el procedimiento a brocha resiste dos ciclos más que sin fibra y con el $1 \%$ resiste hasta 12 ciclos. El procedimiento a rodillo, en ambas proporciones de fibra, es estable.

TABLA 2

Resultados por soporte y pintura del ensayo de cámara climática (procedimiento: pistola)

\begin{tabular}{|l|c|c|c|}
\hline \multirow{2}{*}{ Soporte/Pintura } & \multicolumn{3}{|c|}{ Procedimiento: pistola } \\
\cline { 2 - 4 } & Sin Fibra & $0,25 \%$ & $0,4 \%$ \\
\hline Mortero/Imperm. & OK & OK & OK \\
\hline Mortero/Plástica & OK & OK & OK \\
\hline Mortero/Temple & OK & OK & OK \\
\hline Cartón-Yeso/Plástica & OK & OK & OK \\
\hline Cartón-Yeso/Temple & OK & OK & OK \\
\hline Yeso/Plástica & 1 ciclo & OK & OK \\
\hline Yeso/Temple & 4 ciclos & 5 ciclos & OK \\
\hline
\end{tabular}


La Tabla 3 presenta los resultados por soporte y pintura. Se ha empleado de nuevo el símbolo "OK" cuando la probeta pasa el ensayo sin que aparezcan daños. En caso de no superar el ensayo se ha especificado el número de ciclo en el que aparece el primer daño.

Se observa que la adición de fibra mejora la resistencia al craquelado para todas las pinturas y procedimientos; es decir, mejora su comportamiento ante ciclos de congelación-descongelación. Además, la fibra no es causa de la modificación del comportamiento de la pintura ante los agentes exteriores.

\subsection{Ensayos de resistencia a la niebla salina}

A continuación se detallan las conclusiones obtenidas por pintura y soporte, teniendo en cuenta que en este ensayo solamente se han realizado pruebas sobre muestras con los procedimientos de brocha y rodillo.

La pintura al temple ha demostrado ser más estable con la adición de fibra sobre cualquiera de los soportes ensayados cuando se ha utilizado el procedimiento de rodillo. Sobre yeso y yeso laminado se han obtenido buenos resultados con las proporciones de $0,5 \%$ y $1 \%$ en peso, mientras que en el caso del soporte de mortero los resultados son mejores con el $0,5 \%$ de fibra.

La pintura plástica también se ha mostrado más estable sobre los tres soportes con la adición de fibra. Sobre yeso se han obtenido los mejores resultados con el $0,5 \%$ de fibra, mientras que sobre yeso laminado y mortero los resultados son buenos tanto con el $0,5 \%$ como con el $1 \%$. En este último soporte, el procedimiento aconsejado es el rodillo.

La pintura de impermeabilización sobre mortero se vuelve igualmente más estable con la adición de fibra, siendo mejor con el $1 \%$ de fibra, tanto en brocha como en rodillo.
En la Tabla 4 se indican aquellas muestras que mejoraron los resultados respecto a la pintura sin fibra en este ensayo.

\subsection{Otros ensayos}

Determinación del espesor de película. En la Figura 4 se han representado gráficamente el espesor de película medido para una pintura al temple sobre mortero sin fibra y los espesores de película que se obtienen cuando la pintura se ha reforzado con distintas proporciones de hilos de diferentes longitudes. Se han representado también los datos obtenidos para distintos procedimientos de aplicación. El convenio de símbolos que se ha utilizado es el descrito en el epígrafe 4.1.

La Figura 4 permite deducir que no existe una variación apreciable de espesor de película entre las pinturas sin reforzar y las pinturas reforzadas con hilos de $3 \mathrm{~mm}$ de longitud estando las variaciones observadas mas relacionadas con el procedimiento utilizado y el usuario que la aplica que con la presencia de fibra.

A pesar de que la incorporación de vidrio a la pintura podría producir una variación en el $\mathrm{pH}$, aumentando su acidez, los datos obtenidos en el ensayo de medición de esta propiedad indican que no cambió sustancialmente con la adición de fibra. Los datos de variación obtenidos entre la pintura con y sin fibra no pasaron de 10 décimas en todos los procedimientos.

En cuanto a la viscosidad del producto, hay que tener en cuenta que cuanto mayor sea el valor de ésta, más dificil será aplicarlo, por lo que el coste sería mayor. Sin embargo, en los ensayos realizados se observó que los valores de la viscosidad no variaron sustancialmente con ninguna de las proporciones de fibra añadidas; e incluso se obtuvo una disminución con la adición de fibra en la pintura al temple, en las dos proporciones empleadas.

TABLA 3

Resultados por soporte y pintura del ensayo de cámara climática (procedimiento: brocha y rodillo)

\begin{tabular}{|l|c|c|c|c|c|c|}
\hline \multirow{2}{*}{ Soporte/Pintura } & \multicolumn{3}{|c|}{ Procedimiento: brocha } & \multicolumn{3}{c|}{ Procedimiento: rodillo } \\
\cline { 2 - 7 } & $\begin{array}{c}\text { Sin } \\
\text { Fibra }\end{array}$ & $0,5 \%$ & $1 \%$ & Sin Fibra & $0,5 \%$ & \\
\hline Mortero/Impcrm. & 1 ciclo & 3 ciclos & 12 ciclos & 1 ciclo & OK & OK \\
\hline Mortero/Plástica & OK & OK & OK & 12 ciclos & OK & OK \\
\hline Mortero/Temple & $\begin{array}{c}8 \\
\text { ciclos }\end{array}$ & 9 ciclos & OK & 5 ciclos & OK & OK \\
\hline Cartón-Yeso/Plástica & OK & OK & OK & 11 ciclos & 13 ciclos & OK \\
\hline Cartón-Yeso/Temple & $\begin{array}{c}5 \\
\text { ciclos }\end{array}$ & 6 ciclos & OK & 4 ciclos & 13 ciclos & OK \\
\hline Yeso/Plástica & $\begin{array}{c}2 \\
\text { ciclos }\end{array}$ & 3 ciclos & OK & 3 ciclos & 4 ciclos & OK \\
\hline Yeso/Tcmplc & 1 ciclo & 2 ciclos & 3 ciclos & 1 ciclos & 2 ciclos & 3 ciclos \\
\hline
\end{tabular}


TABLA 4

Resultados por soporte y pintura del ensayo de niebla salina (procedimiento: brocha y rodillo)

\begin{tabular}{|c|c|c|c|c|c|c|}
\hline \multirow[b]{2}{*}{ Soporte/Pintura } & \multicolumn{3}{|c|}{ Procedimiento: brocha } & \multicolumn{3}{|c|}{ Procedimiento: rodillo } \\
\hline & $\begin{array}{c}\text { Sin } \\
\text { Fibra }\end{array}$ & $0,5 \%$ & $1 \%$ & Sin Fibra & $0,5 \%$ & $1 \%$ \\
\hline Mortero/Imperm. & & & $\nabla$ & & & $\nabla$ \\
\hline Mortero/Plástica & & $\nabla$ & & & $\nabla$ & $\nabla$ \\
\hline Mortero/Temple & & & & & $\nabla$ & \\
\hline Cartón-Yeso/Plástica & & $\nabla$ & $\nabla$ & & $\nabla$ & $\nabla$ \\
\hline Cartón-Yeso/Temple & & & & & $\nabla$ & $\nabla$ \\
\hline Yeso/Plástica & & $\nabla$ & & & $\nabla$ & \\
\hline Yeso/Temple & & & & & $\nabla$ & $\nabla$ \\
\hline
\end{tabular}

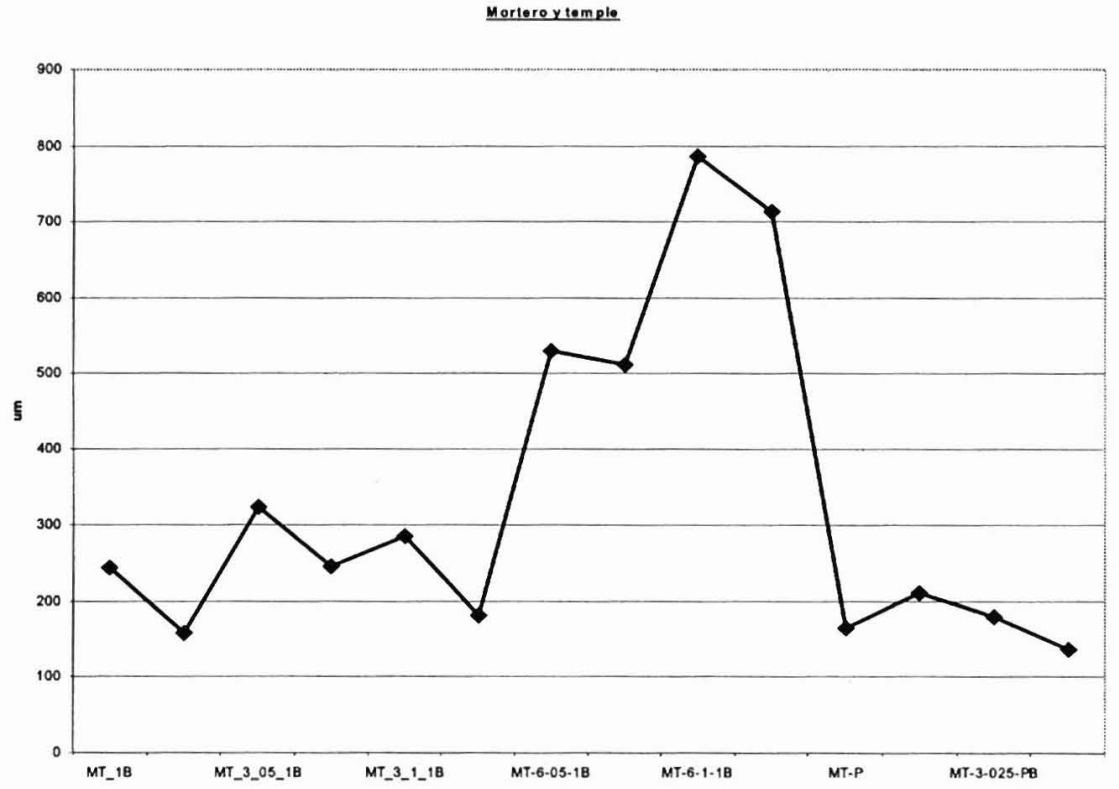

Figura 4.- Ejemplo de gráfico de espesor de pelicula.

Los ensayos colorimétricos y de brillometría han demostrado que la adición de hilos cortos de fibra de vidrio de $3 \mathrm{~mm}$ no ha alterado las propiedades intrínsecas de las pinturas como son el color y el brillo.

Los resultados obtenidos en el ensayo de resistencia del color realizado en la cámara Xenotest, permiten afirmar que las fibras no han alterado la apariencia de las superficies de la pintura en ninguna de las técnicas.

\section{CONCLUSIONES}

Los resultados obtenidos en los ensayos permiten concluir que la adición de fibras de vidrio de característica dispersable a los tipos de pintura que se han ensayado (temple, plástica y de impermeabilización), aplicadas sobre los soportes estudiados, mejora las propiedades del producto: su durabilidad, su resistencia a niebla salina y su resistencia al cuarteo en ciclos de congelacióndescongelación.

Además, la adición de fibras de vidrio no ha alterado negativamente otras propiedades intrínsecas de las pinturas, como el color, el brillo, la rugosidad superficial, el pH y la viscosidad de la mezcla.

Tras los trabajos realizados puede afirmarse que el empleo de pinturas reforzadas con fibras de longitud igual o superior a $6 \mathrm{~mm}$ ocasiona diversos tipos de complicaciones durante su empleo. Tras la experiencias realizadas se recomienda utilizar la fibra de $3 \mathrm{~mm}$ de longitud de 
refuerzo, que se ha revelado idónea por sus ratios prestación/aplicación. Para esta longitud de fibra se han determinado las fracciones gravimétricas óptimas y se ha comprobado que el espesor de película permanece prácticamente constante.

- Pinturas aplicadas con pistola:

- La pintura de impermeabilización sobre mortero funciona bien en las dos proporciones estudiadas de $0,25 \%$ y $0,4 \%$ de fibra.

- En las pinturas aplicadas sobre mortero, los mejores acabados se obtienen con el $0,25 \%$ de refuerzo.

- En el resto de los soportes aparecen fibras salientes por lo que se precisa aplicar un acabado con rodillo.

- Para proporciones de refuerzo superiores a un $0,4 \%$ pueden ocasionarse atascos en la pistola y en el émbolo de la bomba.

- Pinturas aplicadas con brocha y rodillo:

- La fibra permite la aplicación de la pintura tanto con brocha como con rodillo sin que haya que modificar las técnicas de ejecución.

- Cuando por razones de durabilidad se emplee una proporción de refuerzo del $1 \%$ de fibra en peso se aconseja un aumento del batido previo.

- Cuando la proporción de fibra seleccionada es del 0,5\% el procedimiento se hace más fácil de extender en las tres pinturas (plástica, de impermeabilización y al temple).

- La adición de las fibras ha de realizarse como última fase del proceso de preparación de las pinturas, esto es, una vez diluidas si procede o preparadas para su uso. El batido de las fibras puede ser manual o por procedimientos mecánicos. En este último caso el batido no debe de ser excesivamente violento y ha de aplicarse durante un tiempo suficiente hasta que se vean las fibras totalmente disueltas (dispersas en filamentos) y sin que aparezcan grumos o acumulaciones que pudieran impedir o perjudicar un proceso normal de puesta en obra de la pintura.

- El aplicador debe hacer hincapié en realizar un procedimiento que garantice la homogeneidad en la distribución de la fibra en la pintura ya que, sobre todo en el procedimiento a brocha, se podría arrastrar la fibra con el útil y preparar las muestras como si no tuvieran refuerzo.

\section{BIBLIOGRAFÍA}

- AAVV, La pintura como material de construcción ${ }_{2}$ Ed.: Akzo Nobel Coatings, S. A. 1998.

- Juan García Castán, Manual de la pintura en la construcción ${ }_{2}$ Barcelona, Ed.: Anspi, 1996.

Normas y procedimientos de Ensayo:

- UNE EN 23270, "Pinturas, barnices y sus materias primas Temperaturas y humedades para acondicionamiento y ensayo".

- UNE 48.073.94 Parte 2, Apartado 9.2.

- UNE 48.026.80 Pinturas y barnices.

- UNE 11-019.89 Parte 1 ${ }^{a}$ Brillo Especular.

- UNE 48-025-79. Resistencia a ciclos de enfriamiento.

- UNE 53-235-85. Métodos de exposición a una fuente de luz artificial.

- 11-PEN-MB-20 del Centro Tecnológico de la Madera (Toledo).

- UNE 40-435-84. Diferencias de color según el sistema CIELAB.

- UNE 56.843.01 